\title{
Microwave-induced electric discharges on metal particles for the synthesis of inorganic nanomaterials under solvent-free conditions
}

\author{
Vijay Tripathi ${ }^{\ddagger}$, Harit Kumar ${ }^{\ddagger}$, Anubhav Agarwal and Leela S. Panchakarla ${ }^{*}$
}

\author{
Full Research Paper \\ Address: \\ Department of Chemistry, Indian Institute of Technology Bombay, \\ Powai, Mumbai 400076, India \\ Email: \\ Leela S. Panchakarla* - panchakarla@chem.iitb.ac.in \\ * Corresponding author $\ddagger$ Equal contributors \\ Keywords: \\ electric discharges; microwave synthesis; nanomaterials; transmission \\ electron microscopy
}

\author{
Beilstein J. Nanotechnol. 2020, 11, 1019-1025. \\ doi:10.3762/bjnano.11.86 \\ Received: 03 February 2020 \\ Accepted: 22 June 2020 \\ Published: 13 July 2020 \\ This article is part of the thematic issue "Graphene and beyond". \\ Guest Editor: G. U. Kulkarni \\ (C) 2020 Tripathi et al.; licensee Beilstein-Institut. \\ License and terms: see end of document.
}

\begin{abstract}
Microwave irradiation of metals generates electric discharges (arcs). These arcs are used to generate nanoparticles of $\mathrm{Cu}$ and $\mathrm{Ni}$ and one-dimensional nanorods of $\mathrm{CuS}, \mathrm{ZnF}_{2}$, and $\mathrm{NiF}_{2}$ protected with fluorinated amorphous carbon. We have also synthesized reduced graphene oxide and partially rolled graphene by this method.
\end{abstract}

\section{Introduction}

The synthesis of nanomaterials in short time intervals with fewer chemicals has become increasingly important in materials science. Traditional routes of synthesizing nanomaterials, including sol-gel synthesis, solvothermal synthesis, arc-discharge synthesis, or laser ablation, require either large amounts of chemicals or longer synthesis times, or both [1]. Microwave synthesis has become popular in the last three decades as an alternative route for synthesizing molecules and materials at a significantly shorter time scale [2-8]. Dielectric heating under microwave irradiation both in solution and in the solid state rapidly increases the reaction temperature and helps to improve reaction kinetics significantly. This reduces drastically the reaction time $[9,10]$. Non-thermal effects may also influence the reaction kinetics, which is still a subject of discussion $[9,11]$. Bulk metals generally reflect microwaves, whereas fine metal powders or thin films can couple with microwaves (the penetration depth of microwaves in metals is $1-2 \mu \mathrm{m}$ ). This will quickly increase the temperature through conduction mechanisms, which enables the sintering of metals by using microwaves [12-14]. It was found that the sintering of metal powders by microwaves produces products that are denser than and mechanically superior to the ones obtained by conventional heating [14]. Other than reflection and conduction, metal particles usually produce electric discharges (arcs) when exposed to microwaves due to the formation of high electric field gradients at sharp edges on the metal surfaces [12]. The generation of arcs 
might be the reason why microwave irradiation has not been used to generate different nanomaterials from metal particles. However, some studies show that the treatment of metals under microwave irradiation in organic solvents can carbonize the organic solvents forming carbon-coated metallic nanoparticles $[15,16]$. Recently, Pentsak et al. have shown that metals, such as $\mathrm{Cu}, \mathrm{Fe}$, and $\mathrm{Mo}$, on carbon form nanometer-scale structures under microwave heating [17]. However, the microwave discharge technique, which is fast, solvent-free, and easy to set up technically, has not been explored to its fullest potential to synthesize different nanomaterials with controlled morphology.

In this communication, we report on the microwave-induced electric discharge synthesis of $\mathrm{Cu}, \mathrm{Ni}$, und $\mathrm{Zn}$ nanoparticles from metal particles. Also, we can control the morphology of the nanomaterials, which has not been achieved before. $\mathrm{ZnF}_{2}$, $\mathrm{NiF}_{2}$, and $\mathrm{CuS}$ nanorods covered with amorphous fluorinated carbon were synthesized. We have also extended this procedure to synthesize reduced graphene oxide and graphene without using any solvents or additional surfactants.

\section{Results and Discussion}

Smooth surfaces on commercially available metal particles do not create arcs under microwave irradiation. Instead, they heat up or reflects the microwaves. Thus, activating metal surfaces by acid treatment is essential before using metal particles in further microwave arc experiments. All metal powders were treated with $0.5 \mathrm{M}$ nitric acid under sonication for $10 \mathrm{~min}$ to create rough surfaces. In this process, copper partially gets oxidized to $\mathrm{Cu}_{2} \mathrm{O}$. The X-ray diffraction (XRD) patterns show reflections of $\mathrm{Cu}_{2} \mathrm{O}$ after acid treatment, while the majority of $\mathrm{Cu}$ remains in the metallic form (Figure 1a). Scanning electron microscopy (SEM) images of copper powder before and after acid treatment are shown in Figure 1b and Figure 1c, respectively. The SEM image clearly shows the sharp edges on acidtreated copper. Graphitic carbon nitride $\left(\mathrm{g}-\mathrm{C}_{3} \mathrm{~N}_{4}\right)$ or graphite powder (commercially available) are used as carbon source. $\mathrm{g}-\mathrm{C}_{3} \mathrm{~N}_{4}$ is synthesized and characterized according to [18]. $\mathrm{X}$-ray diffraction (XRD) and X-ray photoelectron spectroscopy (XPS) confirms the formation of $\mathrm{g}-\mathrm{C}_{3} \mathrm{~N}_{4}$ (Figure $\mathrm{S} 1$ in Supporting Information File 1).

The reactions were carried out in quartz or Teflon beakers. A Teflon beaker also served as a carbon and fluorine source for the experiments. Typically, $100 \mathrm{mg}$ of acid-treated metal powder mixed either with graphite powder or $\mathrm{g}_{-} \mathrm{C}_{3} \mathrm{~N}_{4}(50 \mathrm{mg})$ were placed inside a domestic kitchen microwave $(2.54 \mathrm{GHz}$, power $700 \mathrm{~W}$ ) and treated for periods of time between $5 \mathrm{~s}$ and $2 \mathrm{~min}$. Arcs were generated in the activated metal, which evaporated the metal along with carbon. Carbon-coated metallic nanoparticles formed on the top lid of the reaction vessel. For

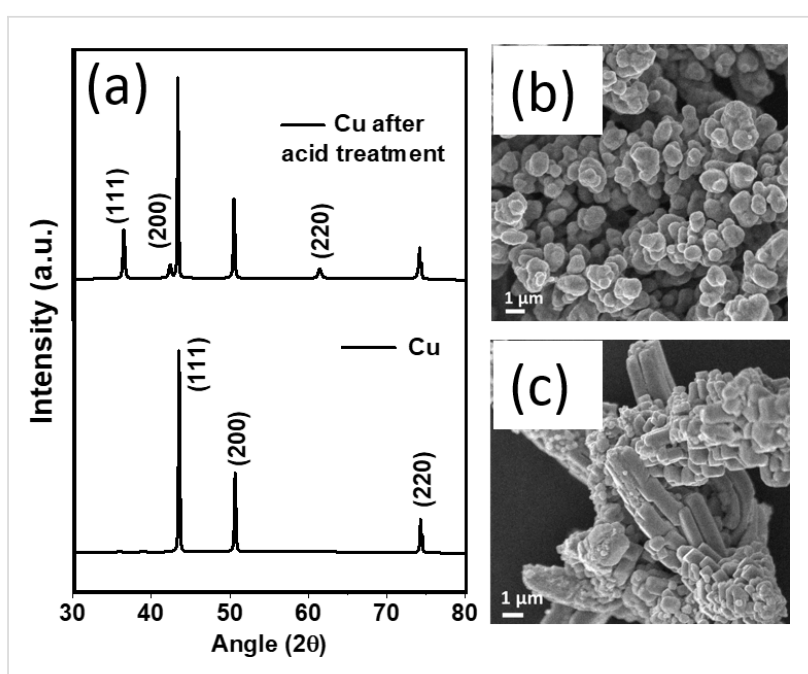

Figure 1: (a) XRD patterns of commercially available Cu powder and Cu powder after treatment with $0.5 \mathrm{M} \mathrm{HNO}_{3}$. (b) SEM images of pure Cu powder and (c) acid-treated Cu powder.

synthesizing nanorods, sulfur was used as a growth promoter. Typically, a mixture of activated metal (100 mg), sulfur powder $(25 \mathrm{mg})$ and $\mathrm{g}-\mathrm{C}_{3} \mathrm{~N}_{4}(50 \mathrm{mg})$ were added to a Teflon beaker and irradiated with microwaves. It is important to note that in the absence of carbon (graphite/g- $\mathrm{C}_{3} \mathrm{~N}_{4}$ ), the arc synthesis yielded a mixture of metal and metal oxide nanoparticles and the particle sizes were found to be difficult to control.

In Figure 2a, we show a schematic of a reaction vessel inside a microwave device. Figure $2 b$ shows the optical image of plasma generated in the reaction vessel during microwave irradiation. Microwave irradiation of activated metals mixed with either graphite or graphitic carbon nitride $\left(\mathrm{g}-\mathrm{C}_{3} \mathrm{~N}_{4}\right)$ yields carboncoated or nitrogen-doped carbon-coated metallic nanoparticles. When these reactions are conducted in a Teflon reactor, the products are further functionalized with fluorine. Figure $3 \mathrm{a}$ shows XRD patterns of $\mathrm{Cu}$ and $\mathrm{Ni}$ nanoparticles generated after 1 min of microwave treatment of activated $\mathrm{Cu}$ and $\mathrm{Ni}$ powders in the presence of $\mathrm{g}-\mathrm{C}_{3} \mathrm{~N}_{4}$. The patterns show pure phases of $\mathrm{Ni}$ and $\mathrm{Cu}$. The formed nanoparticles are covered with fluorinated amorphous carbon. Figure $3 \mathrm{~b}$ shows the SEM image of $\mathrm{Cu}$ nanoparticles covered with amorphous fluorinated carbon with an average size of $80 \mathrm{~nm}$. The $\mathrm{C}-\mathrm{F}$ bonds at the surface of the metallic nanoparticles could be further functionalized for different applications such as drug delivery [19]. A SEM image of $\mathrm{Ni}$ nanoparticles is shown in Figure S2 in Supporting Information File 1. Figure S3 in Supporting Information File 1 shows the SEM images of $\mathrm{Cu}$ nanoparticles covered with amorphous fluorinated carbon synthesized using graphite as carbon source. The structural and morphological features of these $\mathrm{Cu}$ nanoparticles are similar to those of $\mathrm{Cu}$ nanoparticles produced using $\mathrm{g}-\mathrm{C}_{3} \mathrm{~N}_{4}$. In contrast to $\mathrm{Cu}$ and $\mathrm{Ni}$, microwave irradiation of zinc metal in 

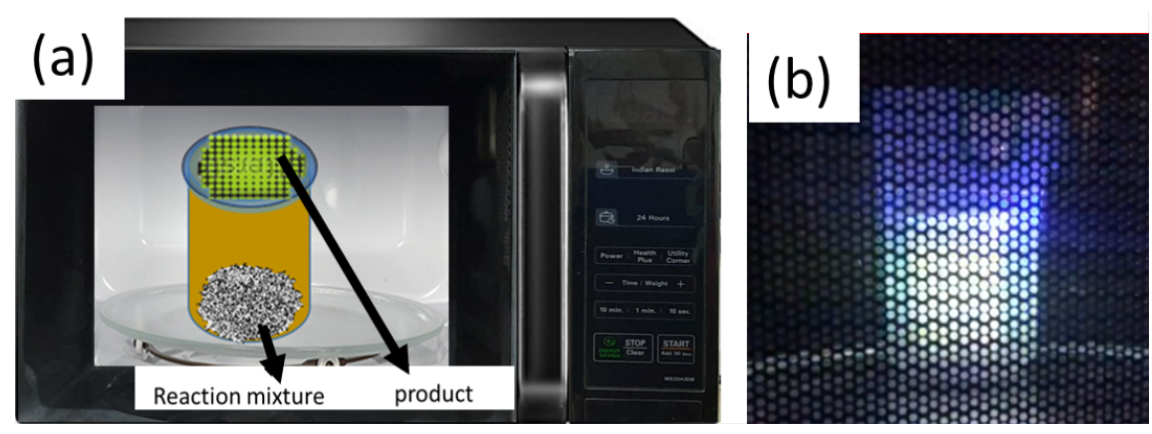

Figure 2: (a) Schematic of the synthesis in a microwave reactor, (b) plasma generated with metal particles under microwave irradiation.

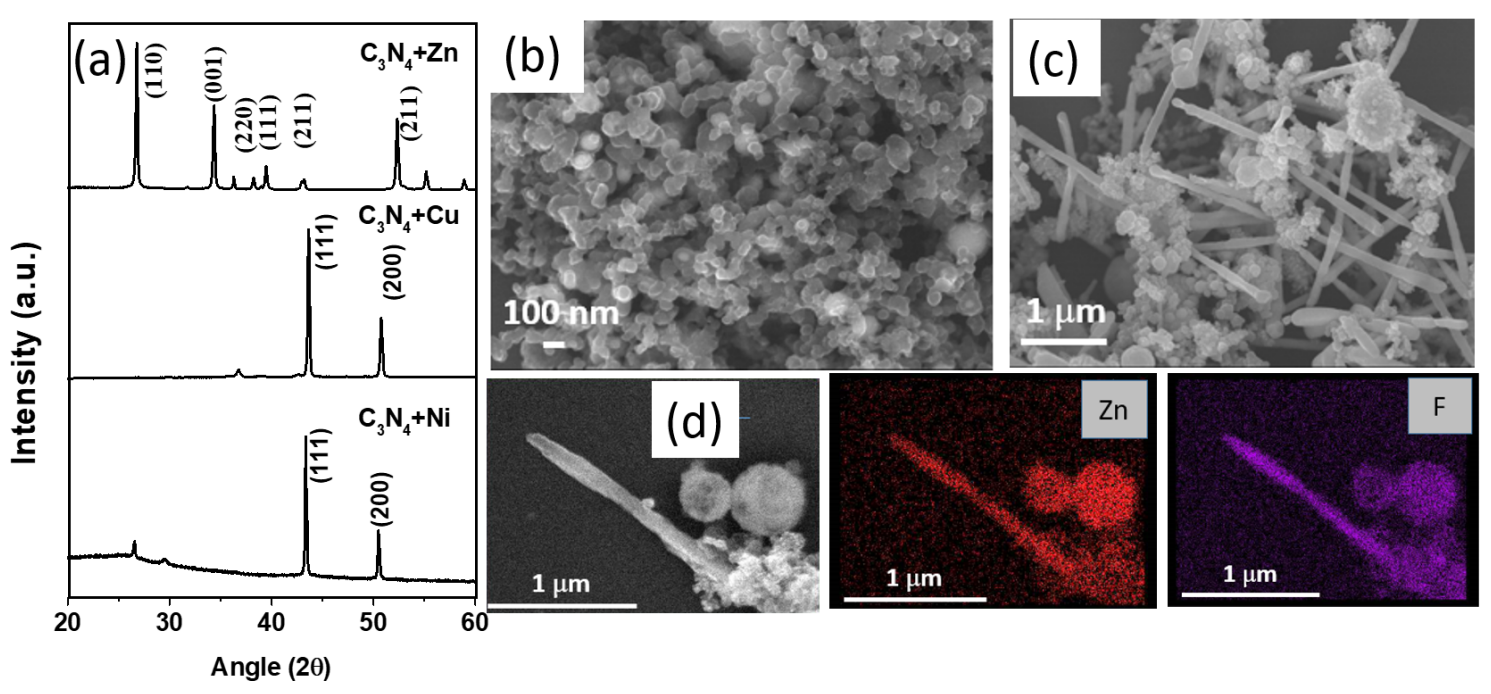

Figure 3: (a) XRD patterns of carbon-coated $\mathrm{Cu}$ and Ni nanoparticles and $\mathrm{ZnF}_{2}$ nanorods. (b) SEM images of carbon-coated Cu nanoparticles. (c) SEM images of $\mathrm{ZnF}_{2}$ nanorods. (d) $\mathrm{SEM}$ image and EDS elemental mapping of a single $\mathrm{ZnF}_{2}$ nanorod.

the presence of Teflon and $\mathrm{g}-\mathrm{C}_{3} \mathrm{~N}_{4}$ creates $\mathrm{ZnF}_{2}$ nanorods inside fluorinated carbon. From XPS (Figure S4, Supporting Information File 1) the carbon-to-fluorine ratio was calculated to be $3: 2 . \mathrm{Zn}$ is highly electropositive and reacts readily with fluorine from Teflon and forms $\mathrm{ZnF}_{2}$. The XRD patterns in Figure $3 \mathrm{a}$ confirm the formation of $\mathrm{ZnF}_{2}$. Both nanorods and nanoparticles of $\mathrm{ZnF}_{2}$ were formed as can be seen from the SEM image (Figure 3c). Figure 3d shows the energy-dispersive spectroscopy (EDS) mapping of $\mathrm{Zn}$ and F, which confirms the presence of $\mathrm{Zn}$ and $\mathrm{F}$ in the nanorod. The average diameter of the nanorods is $100 \mathrm{~nm}$ and the length ranges from 2 to $3 \mu \mathrm{m}$. The formation of external amorphous fluorinated carbon nanotubes helps as a template in the formation of $\mathrm{ZnF}_{2}$ nanorods.

It is well established that sulfur acts as a growth promoter for carbon nanotubes and carbon fibers [20,21]. Thus, sulfur was introduced to the reaction mixtures to improve the yield of nanorods. The microwave treatment of activated $\mathrm{Zn}$ metal with g- $\mathrm{C}_{3} \mathrm{~N}_{4}$ and sulfur in a Teflon container produced $\mathrm{ZnF}_{2}$ nanorods in high yield. The XRD patterns in Figure S5 in Supporting Information File 1 confirm the phase purity of $\mathrm{ZnF}_{2}$. Figure S6 in Supporting Information File 1 shows the SEM and transmission electron microscopy (TEM) images of $\mathrm{ZnF}_{2}$ nanorods produced in the presence of sulfur. The SEM images indicate the high yield of $\mathrm{ZnF}_{2}$ nanorods. The high-resolution TEM (HRTEM) image in Figure S6d (Supporting Information File 1) confirms the single-crystalline nature of $\mathrm{ZnF}_{2}$. Similarly, microwave treatment of $\mathrm{Ni}$ in the presence of sulfur in a Teflon beaker yielded $\mathrm{NiF}_{2}$ nanorods along with $\mathrm{Ni}$ nanoparticles (Figure S5 and Figure S7, Supporting Information File 1). It is important to note that a similar experiment without sulfur yielded only Ni nanoparticles. Figure S7 in Supporting Information File 1 shows SEM and TEM images of $\mathrm{NiF}_{2}$ nanorods. The EDS mapping in Figure S7c (Supporting Information File 1) confirms the presence of $\mathrm{Ni}, \mathrm{F}$ and $\mathrm{C}$ in the $\mathrm{NiF}_{2}$ nanorods. $\mathrm{A}$ TEM image of $\mathrm{NiF}_{2}$ nanorod covered with amorphous carbon is 
shown in Figure S7d (Supporting Information File 1). The HRTEM image (Figure S7e, Supporting Information File 1) clearly shows the single-crystalline nature of the $\mathrm{NiF}_{2}$ nanorod.

Interestingly, microwave treatment of copper in the presence of sulfur in Teflon yielded $\mathrm{CuS}$ nanorods instead of $\mathrm{CuF} / \mathrm{CuF}_{2}$ nanorods. The reactivity of $\mathrm{Cu}$ with sulfur is higher than that with fluorine as soft-soft interactions between $\mathrm{Cu}$ and $\mathrm{S}$ dominate the product stability compared to soft-hard interactions between $\mathrm{Cu}$ and $\mathrm{F}$. The XRD pattern in Figure $4 \mathrm{a}$ confirms the hexagonal covellite structure of CuS. The SEM image in Figure $4 \mathrm{~b}$ and the TEM image in Figure $4 \mathrm{c}$ confirm the onedimensional nature. $\mathrm{CuS}$ nanorods are single-crystalline as can be seen from the HRTEM image in Figure 4d. CuS nanorods were found to grow along the [101] direction. The average core diameter of the CuS nanorods is about $25 \mathrm{~nm}$ and the thickness of the amorphous layer on top of the $\mathrm{CuS}$ nanorods is about $5 \mathrm{~nm}$.

Reports on the generation of inorganic nanomaterials from metals by electric arcs under microwave irradiation are rare. Here, we have shown that metals can effectively interact with microwaves when metallic particles contain rough surfaces or sharp edges. When electrically conducting rough surfaces are exposed to microwaves, electric fields distribute inhomogeneously along the surface of the conductor. At tips and sharp edges, very high electric field gradients occur, leading to the ionization of the material and the surrounding gas, followed by electric discharges [12]. These discharges might lead to the melting of the metal and the evaporation of metal, surrounding carbon, sulfur, and fluorine (from Teflon). A chemical reaction between the metal and sulfur and/or fluorine leads to the formation of metal sulfides and/or fluorides depending on the reactivity. $\mathrm{Cu}$ and $\mathrm{Ni}$, in the presence of graphite and /or g- $\mathrm{C}_{3} \mathrm{~N}_{4}$ and the absence of sulfur produce metallic nanoparticles. This might be due to the reducing capability of carbon at high temperatures preventing the metals from getting oxidized. When sulfur is used in the reaction mixture along with $g-\mathrm{C}_{3} \mathrm{~N}_{4}$, sulfur helps to produce carbon nanotubes instead of carbon nanospheres. The reactivity of sulfur with the end caps of nanotubes does not allow the carbon nanotubes to close. These amorphous carbon nanotubes help as a template to intercalate metal fluorides/metal sulfides. As the local temperatures are very high, metal fluorides and sulfides melt and fill the nanotubes via capillary forces. The liquids solidify as one-dimensional nanorods inside the nanotubes. The usage of $\mathrm{g}-\mathrm{C}_{3} \mathrm{~N}_{4}$, instead of graphite, was found to be beneficial, especially for obtaining high yields of nanorods. However, the choice of
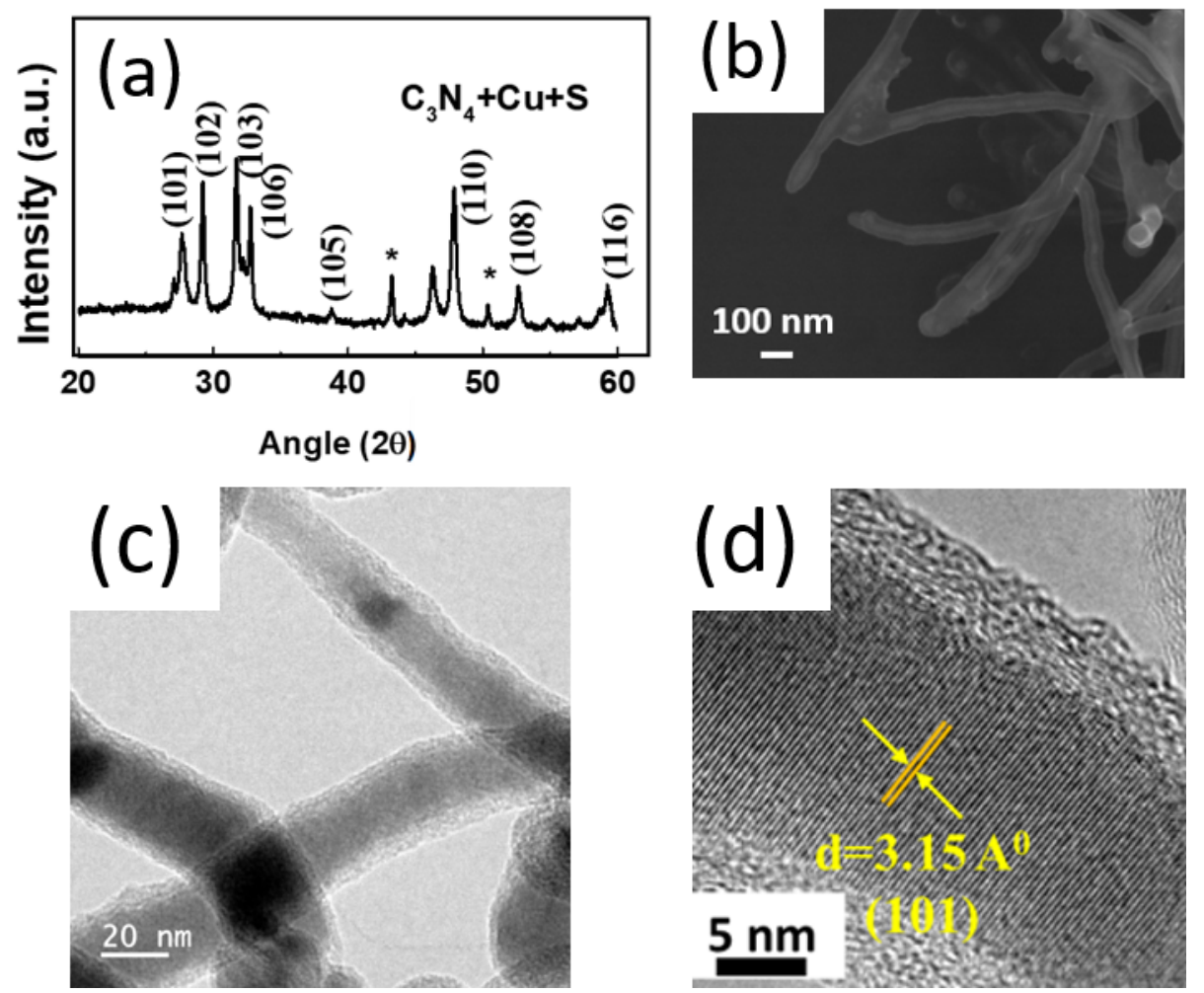

Figure 4: (a) XRD patterns of CuS nanorods synthesized in the presence of sulfur by microwave irradiation of copper in the presence of $g$ - $\mathrm{C}_{3} \mathrm{~N}_{4}$ in a Teflon beaker for 2 min. (b) SEM, (c) TEM and (d) HRTEM images of CuS nanorods. The asterisk (*) in panel (a) indicates the reflections from Cu. 
graphite or $\mathrm{g}-\mathrm{C}_{3} \mathrm{~N}_{4}$ has shown little to no difference in the synthesis of nanoparticles.

We have also studied exfoliation of graphite and graphite oxide (GO) under microwave irradiation in the presence of $\mathrm{Zn}$ metal. We have observed exfoliation of graphite into few-layered graphene. We have also seen a partial rolling of graphene sheets into nanoscrolls (Figure 5a,b). The formation of ultrasmall nanoparticles of $\mathrm{ZnO}$ along with graphene was detected (see Figure S8 in Supporting Information File 1). In the case of GO exfoliation, we have observed the formation of nanosheets of reduced graphite oxide (Figure 5c). These nanosheets still keep hexagonal structure under microwave irradiation as can be seen from the selected area electron diffraction pattern in Figure 5d.

\section{Conclusion}

We have shown that microwave-induced electric discharges on rough metallic surfaces can be effectively used to synthesize nanomaterials with controlled morphology. $\mathrm{Cu}$ and $\mathrm{Ni}$ metallic nanoparticles are stabilized through amorphous carbon. Nanorods of $\mathrm{ZnF}_{2}, \mathrm{NiF}_{2}$ and $\mathrm{CuS}$ are synthesized inside fluorinated amorphous carbon nanotubes in the presence of sulfur. External C-F bonds can be further functionalized readily without disturbing internal materials. We could also produce reduced graphene oxide and graphene partially rolled into nanoscrolls. We hope that this work encourages further research exploring the possibilities to synthesize other inorganic nanomaterials by microwave-induced electric discharge without the need for surfactants and solvents.

\section{Experimental}

\section{Roughening the surface metal powders}

Commercially purchased micrometer-sized metal powders (Ni, $\mathrm{Cu}$ and $\mathrm{Zn}$ ) are treated with nitric acid to create rough surfaces. In a typical reaction, $100 \mathrm{mg}$ of metal powder is transferred to a beaker containing $10 \mathrm{~mL}$ of $0.5 \mathrm{M}$ nitric acid and sonicated for $10 \mathrm{~min}$. The resultant powder is washed with water several times until $\mathrm{pH} 7$ and dried it in an oven at $50{ }^{\circ} \mathrm{C}$ for $2 \mathrm{~h}$ before being used in further microwave experiments.
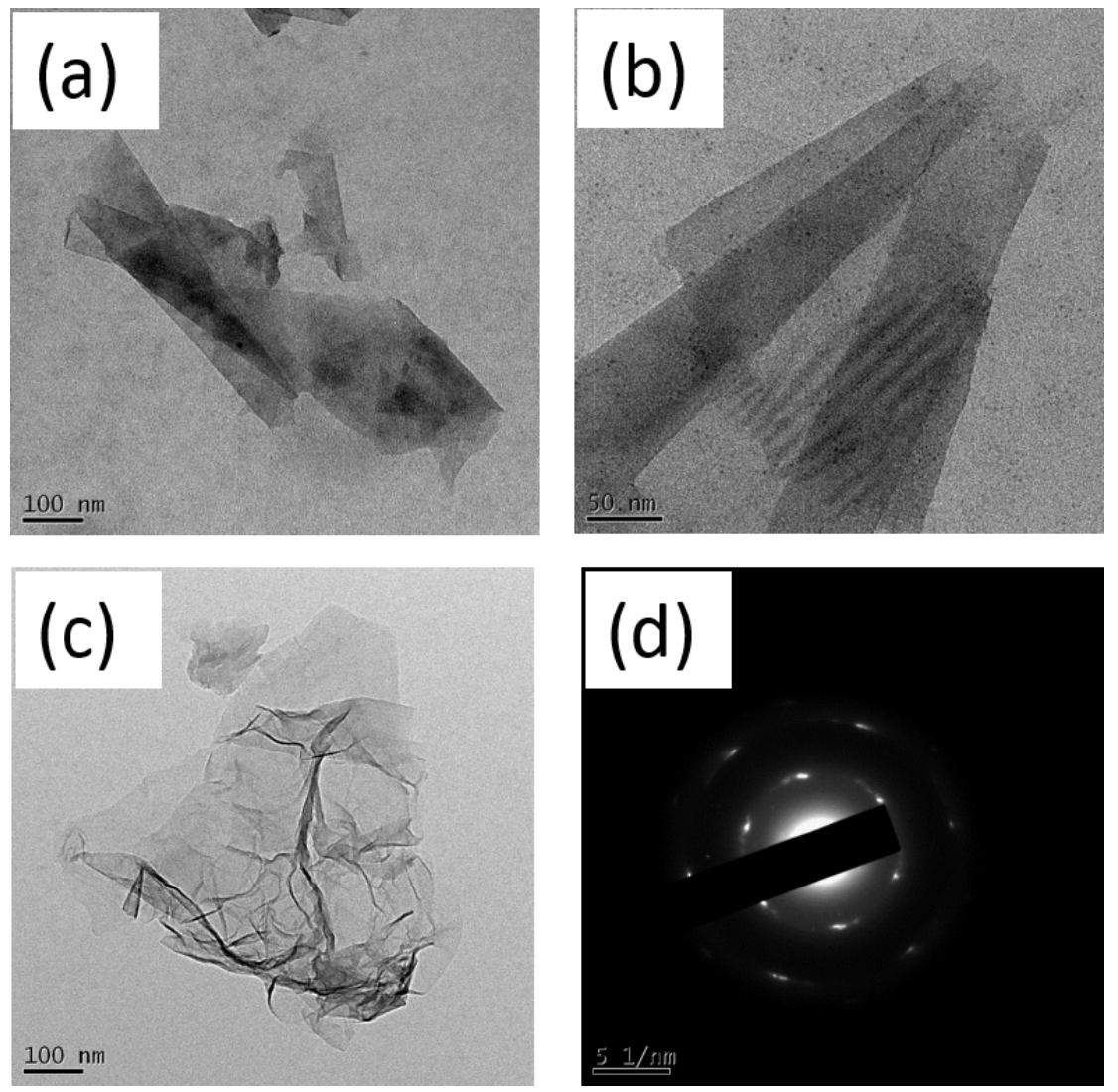

Figure 5: $(a, b)$ TEM images of few-layered graphene partially rolled into nanoscrolls synthesized by irradiating graphite with microwaves in the presence of $\mathrm{Zn}$ metal for $30 \mathrm{~s}$. (c) TEM image of graphite oxide after exfoliation trough microwave irradiation in the presence of $\mathrm{Zn}$ metal for $30 \mathrm{~s}$ and (d) the corresponding selected area electron diffraction pattern. 


\section{Synthesis of graphitic carbon nitride $\left(\mathrm{g}-\mathrm{C}_{3} \mathrm{~N}_{4}\right)$} g- $\mathrm{C}_{3} \mathrm{~N}_{4}$ is synthesized and characterized according to [18]. In a typical reaction, melamine $(150 \mathrm{mg})$ and urea $(71 \mathrm{mg})$ are mixed in a quartz boat and heated at $650{ }^{\circ} \mathrm{C}$ under nitrogen flow for $2 \mathrm{~h}$ to obtain bulk $\mathrm{g}-\mathrm{C}_{3} \mathrm{~N}_{4}$ as orange product.

\section{Generation of nanomaterials using microwave-induced discharge}

To generate nanoparticles by microwave-induced discharge, the reaction is conducted either in a quartz or a Teflon beaker. A Teflon beaker also serves as a carbon and fluorine source for the experiments. Typically, $100 \mathrm{mg}$ of acid-treated metal powder mixed either with graphite powder or $g-\mathrm{C}_{3} \mathrm{~N}_{4}$ $(50 \mathrm{mg})$ and placed inside a domestic kitchen microwave $(2.54 \mathrm{GHz}$, power $700 \mathrm{~W})$ and treated for $5 \mathrm{~s}$ to $2 \mathrm{~min}$. The product containing carbon-coated metallic nanoparticles are collected from the lid of the reaction vessel. For synthesizing nanorods, sulfur is used as a growth promoter. Typically, a mixture of activated metal $(100 \mathrm{mg})$, sulfur powder $(25 \mathrm{mg})$ and $\mathrm{g}_{-} \mathrm{C}_{3} \mathrm{~N}_{4}(50 \mathrm{mg})$ is placed in a Teflon beaker and irradiated with microwaves. The products are collected from the lid of the reaction vessel. The above experiments are performed in air.

\section{Preparation of few-layer graphene and graphite oxide nanosheets}

In a typical experiment, $50 \mathrm{mg}$ of acid-treated $\mathrm{Zn}$ metal is mixed with $100 \mathrm{mg}$ of either graphite or graphite oxide in a quartz beaker and irradiated with microwaves for $1 \mathrm{~min}$. Products are collected from the reaction vessel, sonicated in ethanol for $1 \mathrm{~min}$ and centrifuged for $5 \mathrm{~min}$ at $6000 \mathrm{rpm}$ to separate big metal particles and unreacted graphite. The supernatant solution is collected for further characterization.

\section{Characterization of products}

The reaction products are characterized by X-ray diffraction (PANanalytical X'pert PRO), field-emission scanning electron microscopy (JEOL JSM 7600F, combined with energy-dispersive spectroscopy (EDS)), transmission electron microscopy (JEOL JEM 2100F) and X-ray photoelectron spectroscopy XPS (Thermo VG Scientific MultiLab, ESCA).

\section{Supporting Information}

\section{Supporting Information File 1}

Characterization details of $\mathrm{g}-\mathrm{C}_{3} \mathrm{~N}_{4}$ by XRD and XPS.

Electron microscope analysis of $\mathrm{Ni}, \mathrm{Cu}, \mathrm{ZnF}_{2}, \mathrm{NiF}_{2}$, and

$\mathrm{ZnO}$ nanostructures.

[https://www.beilstein-journals.org/bjnano/content/

supplementary/2190-4286-11-86-S1.pdf]

\section{Acknowledgements}

Authors acknowledge SAIF, NCPRE and IRCC at IIT Bombay for central facilities.

\section{Funding}

LSP acknowledges IIT Bombay and DST-SERB (EMR/2016/ 003594) for financial support. VT thanks CSIR for a fellowship.

\section{ORCID ${ }^{\circledR}$ iDs}

Leela S. Panchakarla - https://orcid.org/0000-0001-5829-3377

\section{Preprint}

A non-peer-reviewed version of this article has been previously published as a preprint doi:10.3762/bxiv.2020.14.v1

\section{References}

1. Rao, C. N. R.; Müller, A.; Cheetham, A. K., Eds. The Chemistry of Nanomaterials: Synthesis and Applications; Wiley-VCH: Weinheim, Germany, 2004. doi:10.1002/352760247x

2. Kitchen, H. J.; Vallance, S. R.; Kennedy, J. L.; Tapia-Ruiz, N.; Carassiti, L.; Harrison, A.; Whittaker, A. G.; Drysdale, T. D.; Kingman, S. W.; Gregory, D. H. Chem. Rev. 2014, 114, 1170-1206. doi:10.1021/cr4002353

3. Ni, Z.; Masel, R. I. J. Am. Chem. Soc. 2006, 128, 12394-12395. doi:10.1021/ja0635231

4. Polshettiwar, V.; Varma, R. S. Acc. Chem. Res. 2008, 41, 629-639. doi:10.1021/ar700238s

5. Mallikarjuna, N. N.; Varma, R. S. Cryst. Growth Des. 2007, 7, 686-690. doi:10.1021/cg060506e

6. Bilecka, I.; Niederberger, M. Nanoscale 2010, 2, 1358-1374. doi:10.1039/b9nr00377k

7. Liu, Z.; Wang, J.; Kushvaha, V.; Poyraz, S.; Tippur, H.; Park, S.; Kim, M.; Liu, Y.; Bar, J.; Chen, H.; Zhang, X. Chem. Commun. 2011, 47, 9912-9914. doi:10.1039/c1cc13359d

8. Bajpai, R.; Wagner, H. D. Carbon 2015, 82, 327-336. doi:10.1016/j.carbon.2014.10.077

9. Dudley, G. B.; Richert, R.; Stiegman, A. E. Chem. Sci. 2015, 6, 2144-2152. doi:10.1039/c4sc03372h

10. Ching Lau, C.; Kemal Bayazit, M.; Reardon, P. J. T.; Tang, J. Chem. Rec. 2019, 19, 172-187. doi:10.1002/tcr.201800121

11. Díaz-Ortiz, Á.; Prieto, P.; de la Hoz, A. Chem. Rec. 2019, 19, 85-97. doi:10.1002/tcr.201800059

12. Sun, J.; Wang, W.; Yue, Q. Materials 2016, 9, 231. doi:10.3390/ma9040231

13. Whittaker, A. G.; Mingos, D. M. P. J. Chem. Soc., Dalton Trans. 1992, 2751-2752. doi:10.1039/dt9920002751

14. Roy, R.; Agrawal, D.; Cheng, J.; Gedevanishvili, S. Nature 1999, 399 , 668-670. doi:10.1038/21390

15. Hsin, Y.-L.; Lin, C.-F.; Liang, Y.-C.; Hwang, K. C.; Horng, J.-C.; Ho, J.-a. A.; Lin, C.-C.; Hwu, J. R. Adv. Funct. Mater. 2008, 18, 2048-2056. doi:10.1002/adfm.200701407

16. Chen, W.; Gutmann, B.; Kappe, C. O. ChemistryOpen 2012, 1, 39-48. doi:10.1002/open.201100013

17. Pentsak, E. O.; Cherepanova, V. A.; Sinayskiy, M. A.; Samokhin, A. V.; Ananikov, V. P. Nanomaterials 2019, 9, 19. doi:10.3390/nano9010019 
18. Dante, R. C.; Martín-Ramos, P.; Correa-Guimaraes, A.; Martín-Gil, J. Mater. Chem. Phys. 2011, 130, 1094-1102.

doi:10.1016/j.matchemphys.2011.08.041

19. Khabashesku, V. N.; Billups, W. E.; Margrave, J. L. Acc. Chem. Res. 2002, 35, 1087-1095. doi:10.1021/ar020146y

20. Zhang, L.; Hou, P.-X.; Li, S.; Shi, C.; Cong, H.-T.; Liu, C.; Cheng, H.-M. J. Phys. Chem. Lett. 2014, 5, 1427-1432. doi:10.1021/jz500419r

21. Suzuki, S.; Mori, S. Chem. Phys. Lett. 2018, 709, 1-6. doi:10.1016/j.cplett.2018.08.037

\section{License and Terms}

This is an Open Access article under the terms of the Creative Commons Attribution License

(http://creativecommons.org/licenses/by/4.0). Please note that the reuse, redistribution and reproduction in particular requires that the authors and source are credited.

The license is subject to the Beilstein Journal of

Nanotechnology terms and conditions:

(https://www.beilstein-journals.org/bjnano)

The definitive version of this article is the electronic one which can be found at: doi:10.3762/bjnano.11.86 